\title{
36. COMMISSION DE LA SPECTROPHOTOMÉTRIE
}

\section{President: M. H. H. Plasketr, Director of the University Observatory, Oxford.}

Membres: MM. J. Baillaud, Beals, C. R. Davidson, Dufay, Dunham, Eddington, Elvey, Fabry, Greaves, Hogg, Kienle, Lindblad, Menzel, Milne, Minnaert, Ohman, Pannekoek, Mme Payne Gaposchkin, MM. Redman, Rosseland, Russell, Sampson, Struve, Tiercy, Unsöld, Woolley.

The present report is the first for which this newly-formed Commission has been responsible. In view of this fact, and in view of the still exploratory nature of many investigations in spectrophotometry, as well as the need for the highest measure of individuality in the attack of the not simple problems involved, it would be premature to propose, simple though it might be to do so, any far reaching plans for co-operative schemes of investigation. These undoubtedly will play a part in the later work of the Commission, but what appears to be needed now is a closer definition of the aims of spectrophotometry, and at least a reference to the many branches of the subject where investigation is needed. The present report attempts to deal with these topics in three successive sections, concerned in turn with the unique property of spectrophotometric measures, the fields of application of spectrophotometry, and recent developments in a still incomplete and difficult technique.

\section{THE UNIQUE PROPERTY OF SPECTROPHOTOMETRIC MEASURES}

I. The object of all measures of radiation is a determination of the radiative characteristics of the body under examination, entirely freed from circumstances peculiar to the method of observation. When the body concerned is an astronomical body, the radiation of which has to be measured after transmission through the terrestrial atmosphere, this object can only be attained if the radiation is subjected to a preliminary spectroscopic analysis, and the flux measured within a wave-length interval $\lambda$ to $\lambda+d \lambda$. A moment's consideration will show why this is so.

Consider for simplicity an astronomical body of circular projected cross section of linear radius $r$ at a distance $R$ in transparent space. If $J \lambda d \lambda$ is the surface brightness of the body within the wave-length interval $\lambda$ to $\lambda+d \lambda$, so that $J_{\lambda} d \lambda d \omega$ is the flux emitted per unit projected area within the solid angle $d \omega$, then $\dot{\phi}_{\lambda}(t) d \lambda$, the flux per unit area measured in the focal plane of a telescope of aperture $A$, focal length $F$, is

$$
\phi_{\lambda}(t) d \lambda=\frac{A}{F^{2}} \alpha_{\lambda} a_{\lambda}^{\prime} J_{\lambda} d \lambda,
$$

where $\alpha_{\lambda}$ is the transmission coefficient of the telescope, $a_{\lambda}$ is the transmission coefficient of the terrestrial atmosphere, and $t(\doteq \sec z)$ is the air mass at the time of observation. If the astronomical body is so remote that it does not present a visible disc, then in the focal plane of the telescope we can measure a total flux

$$
\Phi_{\lambda}(t) d \lambda=A \frac{\pi r^{2}}{R^{2}} \alpha_{\lambda} a_{\lambda}^{t} \bar{J}_{\lambda} d \lambda
$$

where $\bar{J}_{\lambda}$ is the average surface brightness. Provided now we make our flux measurement over a wave-length interval $d \lambda$ so small that $a_{\lambda}$, the transmission coefficient of the earth's atmosphere, is constant, then two determinations of $\phi_{\lambda}(t) d \lambda$ (or 
$\left.\Phi_{\lambda}(t) d \lambda\right)$ at different air masses $t$ suffice to determine the unknown $a_{\lambda}$ and to leave the residual $\frac{A}{F^{2}} \alpha_{\lambda} J_{\lambda} d \lambda$ (or $A \frac{\pi r^{2}}{R^{2}} \alpha_{\lambda} \bar{J}_{\lambda} d \lambda$ ). A final determination of $\alpha_{\lambda}$ for the wavelength interval concerned then yields $J_{\lambda} d \lambda$ (or $\frac{\pi r^{2}}{R^{2}} \bar{J}_{\lambda} d \lambda$ ), the required radiative characteristic of the body itself freed from all effects of observation.

The circumstances are very different, however, if instead of making a spectrophotometric measure $\phi_{\lambda}(t) d \lambda$, the flux is integrated over a wave-length interval within which there is a perceptible variation of $a_{\lambda}$. For simplicity suppose such a measure to be made with an equal energy receiver (the circumstances are not essentially different, from the present point of view, if the integration is performed with a non-uniform receiver, such as the eye or the photographic plate), so that we now measure

$$
\phi(t)=\int_{\lambda_{1}}^{\lambda_{z}} \phi_{\lambda}(t) d \lambda=\frac{A}{F^{2}} \int_{\lambda_{1}}^{\lambda_{z}} \alpha_{\lambda} a_{\lambda}^{t} J_{\lambda} d \lambda
$$

Now we have no longer, as in equation $(I), \phi(t)$ as a simple exponential function of the air mass, and consequently there is no definite method by which a long range extrapolation to air mass zero can be carried out. Indeed in the determination of radiometric magnitudes (as also in visual and photographic magnitudes) all that is attempted is a short range extrapolation to air mass unity, and even this limited empirical extrapolation is attended with uncertainty, because for each body with its characteristic energy distribution, $J_{\lambda}$, a different variation of $\phi(t)$ with $t$ must exist. When, however, this limited extrapolation has been carried out we are still left with $\frac{A}{F^{2}} \int_{\lambda_{1}}^{\lambda_{2}} \alpha_{\lambda} a_{\lambda} J_{\lambda} d \lambda$ as our observed quantity, and if we are to pass to that measured quantity $\int_{\lambda_{1}}^{\lambda_{2}} J_{\lambda} d \lambda$, which is independent of the circumstances of observation, we can only do so by a separate spectrophotometric determination of both $\alpha_{\lambda}$ and $a_{\lambda}$ (the transmission coefficient for air mass unity). In the application of radiometric (as well as visual and photographic magnitudes) the attempt is sometimes made to avoid this difficulty by taking the ratio of the quantity $\int_{\lambda_{1}}^{\lambda_{2}} \alpha_{\lambda} a_{\lambda} J_{\lambda} d \lambda$ for the astronomical body concerned to the same quantity measured for the sun, the idea being to eliminate the unknowns $\alpha_{\lambda}, a_{\lambda}$ and so obtain $\int_{\lambda_{1}}^{\lambda_{3}} J_{\lambda}(*) d \lambda / \int_{\lambda_{1}}^{\lambda_{2}} J_{\lambda}(\odot) d \lambda$. Clearly, however, the unknowns $\alpha_{\lambda}, a_{\lambda}$ will not be eliminated unless $J_{\lambda}(*)$, apart from a constant factor, is identical with $J_{\lambda}(\odot)$. The above arguments apply, and the same difficulty exists, if one works with the flux $\Phi_{\lambda}(t)$, which alone is measurable for a star, instead of the flux $\phi_{\lambda}(t)$.

In short, if the radiative characteristics of astronomical bodies, which have to be observed through the terrestrial atmosphere, are desired, spectrophotometric measures, and spectrophotometric measures alone, will give the desired characteristics freed from all the circumstances of observation. It is for these reasons that the monochromatic magnitudes, now in course of determination at Göttingen and Stockholm, are so important, and that a co-operative scheme of monochromatic magnitude determination may ultimately play a large part in the work of this Commission. 


\section{THE APPLICATION OF SPECTROPHOTOMETRIC MEASURES}

2.I. Continuous Spectrum. Spectrophotometric measures of the flux received in the focal plane of the collecting telescope from a star yield directly from equation ( $\mathrm{I}$ ) its surface brightness, $J_{\lambda} d \lambda$, or from equation (2) $\frac{\pi r^{2}}{R^{2}} \bar{J}_{\lambda} d \lambda$, its flux received per unit area outside the earth's atmosphere (the illumination). In the latter case a subsidiary measurement of angular diameter gives directly the average surface brightness, $\bar{J}_{\lambda} d \lambda$. These measures of $J_{\lambda} d \lambda$, or $\frac{\pi r^{2}}{R^{2}} \bar{J}_{\lambda} d \lambda$, may be tabulated or plotted as a function of wave-length, and such tables or graphs tell the whole story of the radiative characteristics of the star. It is convenient, however, to be able to represent these characteristics for a given star by a single parameter, and that parameter is conveniently called a "temperature." This "temperature" may be defined in three different ways by Kirchhoff's law.

Kirchhoff's law states that the surface brightness of a body emitting temperature radiation is less than, or at best equal to, the surface brightness of a black body maintained at the same temperature. We have

$$
J_{\lambda}=A_{\lambda} B_{\lambda}=A_{\lambda} \frac{2 h c^{2}}{\lambda^{5}}\left(e^{h c / k \lambda T}-\mathrm{I}\right)^{-1},
$$

where $A_{\lambda}(\leqq I)$ is the absorptive power, and $B_{\lambda}$ is the surface brightness of the black body, given by Planck's law as a function of temperature and wave-length on the right-hand side of the equation. The three temperatures which may be defined by equation (4) are the "brightness temperature," the "effective temperature" and the "colour temperature."

The "brightness temperature," $T_{b}(\lambda)$, is defined to be the temperature of a black body with the same surface brightness at the wave-length $\lambda$ as the radiator concerned. That is from (4)

$$
J_{\lambda}=A_{\lambda} \frac{2 h c^{2}}{\lambda^{5}}\left(e^{h c / k \lambda T}-\mathrm{I}\right)^{-1}=\frac{2 h c^{2}}{\lambda^{5}}\left(e^{h c / k \lambda T_{b}(\lambda)}-\mathrm{I}\right)^{-1},
$$

from which it follows, since $A_{\lambda} \leqq \mathrm{I}$, that $T_{b}(\lambda) \leqq T$. In other words the "brightness temperature" is always a lower limit to the true temperature.

Closely similar to the "brightness temperature," and, like it, requiring the surface brightness of the radiator, is the "effective temperature." Integrating equation (4) with respect to wave-length we obtain

$$
J=\int_{0}^{\infty} J_{\lambda} d \lambda=\int_{0}^{\infty} A_{\lambda} B_{\lambda} d \lambda=A \int_{0}^{\infty} B_{\lambda} d \lambda=A \frac{\boldsymbol{\sigma}}{\pi} T^{\mathbf{4}},
$$

where $\sigma$ is Stefan's constant, and $A$ is a mean of $A_{\lambda}$ weighted according to the Planckian function $B_{\lambda}$; since $A_{\lambda} \leqq \mathrm{I}$, it follows that $A \leqq \mathrm{I}$. The "effective temperature" is defined to be the temperature of a black body with the same surface brightness in total radiation as the radiator concerned. That is from (5)

$$
J=A \frac{\sigma}{\pi} T^{4}=\frac{\sigma}{\pi} T_{e}^{4},
$$

from which it follows, since $A \leqq \mathrm{I}$, that $T_{e} \leqq T$. Like the "brightness temperature" the "effective temperature" is always a lower limit to the true temperature, but 
unlike the "brightness temperature" the "effective temperature" has a very limited range of usefulness as applied to astronomical radiators. This immediately follows, since the earth's atmosphere, apart from a small gap at $\lambda 2100$, is completely opaque to radiation to the violet of $\lambda 3000$. Since for stars of all temperatures a definite and unknown fraction of the stellar radiation lies to the violet of $\lambda 3000$, a fraction which for high temperature stars is probably of the order of one-half, it follows that a measure of total stellar radiation can never be made, but that only an estimate based on a long range extrapolation of the energy curve $J_{\lambda}$ can be given. The "effective temperature," defined in terms of total radiation, is therefore not an observable, and for high temperature stars not even a certainly inferable, quantity; the "effective temperature" is not, therefore, a satisfactory parameter for representing the observed radiative characteristics of a star.

For stars of unknown angular diameter spectrophotometric measures give only the quantity $\frac{\pi r^{2}}{R^{2}} \bar{J}_{\lambda} d \lambda$. In this case, since $\frac{r^{2}}{R^{2}}$ is common to measures at all wavelengths in the one star, it follows that the ratio of the surface brightnesses at two wave-lengths may be obtained. We thus have from (4),

$$
\frac{\bar{J}_{l}}{\bar{J}_{\lambda}}=\frac{A_{l}}{A_{\lambda}} \cdot \frac{\lambda^{5}}{l^{5}} \frac{e^{h c / k \lambda T}-\mathrm{I}}{e^{h c / k l T}-\mathrm{I}}=\frac{\lambda^{5}}{l^{5}} \frac{e^{h c / k \lambda T_{c}-\mathrm{I}}}{e^{h c / k l T_{c-I}}} .
$$

We now define the "colour temperature," $T_{c}$, to be the temperature of a black body with the same surface brightness ratio at the two wave-lengths as the star concerned. We thus find from (6) that $T_{e} \leqq T$, according as $A_{c} \doteq A_{\lambda}$ for $\lambda<l$. There is thus no simple relation between "colour temperature" and true temperature.

2.II. The significant feature of the measured surface brightness, $J_{\lambda}$, is not, however, its parametric representation by "brightness and colour temperatures." From the theory of stellar atmospheres, due in its main outlines to the classical investigations of Schwarzschild and Milne, we know that the surface brightness of the emergent radiation bears an intimate relation to the whole structure and composition of that atmosphere. Thus in the simplest possible case the surface brightness in a direction $\theta$ with the outgoing normal is given by

$$
J_{\lambda}(\theta)=K T_{0}^{5} \alpha^{5} \int_{0}^{\infty} \frac{e^{-y} d y}{e^{\alpha(1+p y)-1 / 4}-\mathrm{I}},
$$

where $K$ is a known constant, $T_{o}$ is the boundary temperature (true) of the star, $\alpha=h c / k \lambda T_{0}$, and $p=\frac{3}{2} \cos \theta / n_{\lambda}$. Here $n_{\lambda}$ is the ratio of the mass coefficient of absorption at the wave-length $\lambda$ to the Rosseland mean mass coefficient, the ratio having been assumed constant through the atmosphere in the derivation of the above expression. If this assumption and the other assumptions of local thermodynamic equilibrium, temperature distribution corresponding to pure radiative equilibrium, and absence of continuous scattering, were true of stellar atmospheres, then the representation of the observed surface brightness would be reduced to that of finding appropriate values of $T_{o}$ and $n_{\lambda}$. Thus the surface brightness of the sun has been satisfactorily represented by $T_{o}=4830^{\circ} \mathrm{K}$, and $n_{\lambda}=\mathrm{I}$, and now investigations are in progress as to the atomic absorption coefficients and the chemical composition of the atmosphere which would give rise to such a grey coefficient of absorption. Whether, however, it is in terms of the idealized model of equation (7), or of some more complicated model, that measures of stellar surface brightness in the continuous spectrum are represented, is not at the moment material; the 
essential thing is that it is in such connection that these measures are important, and not in connection with the determination of "brightness and colour temperature."

2 . I2. In the case of extended objects such as globular clusters, and galactic and extra galactic nebulae, spectrophotometric measures yield the surface brightness directly from equation (I). Such measures of surface brightness, not necessarily requiring a large telescope, show every promise of providing a fruitful field for future investigation. Thus, if similarly built nebulae or clusters may be assumed to have identical surface brightness, it follows from (I) that in transparent space such objects should have the same measured surface brightness at the earth. Any departure from this condition would be due, if the not unreasonable initial assumption were true, to absorption of light in space, and such measures of surface brightness would afford the most direct and accurate method of determining that absorption. Again, measures of surface brightness, as a function of position on the nebular image, will yield from ten Bruggencate's analysis* the radial distribution of intensity in elliptical nebulae, and therefore a closer insight into their physical nature. Again, the exact determination of surface brightness as a function of wavelength for the nearer extra galactic nebulae is an essential pre-requisite for determining the magnitude correction to be applied to those nebulae showing large red-shift, and hence is bound to play an important part in finding the spatial distribution of these remote nebulae, possibly in consequence furnishing an observational check that red-shift is due to recessional velocity.

2*2. Line Spectra. Given a knowledge of the atomic absorption coefficient and the mode of formation of the absorption line in the solar or stellar atmosphere, spectrophotometric measures of line profiles yield directly the population of atoms in the various stationary states. From this information "excitation temperatures" and electron pressures may be derived. In no one of its aspects, however, is the interpretation of the measured line profile a simple matter, and two tendencies, depending upon the use of the actual profile, or the equivalent breadth (total absorption) may be noted.

The detailed study of the line profile, and its dependence upon the atomic absorption coefficient, initiated by Unsöld in 1927 , is made difficult, but none the less informative, because the profile is so sensitive an indicator of the mode of formation of the absorption line in the stellar atmosphere. This is well illustrated by the change in profile over the disc of the sun. Future progress in the detailed study of the line profile will depend in part on finding a satisfactory interpretation of the large central intensities of solar absorption lines, and in part, possibly, on numerical integrations of the type foreshadowed by ten Bruggencate $f$ and Pannekoek integrations which yield directly the electron pressure as a function of optical depth. The determination of the highly accurate solar line profiles needed for this type of work should also yield evidence of unsymmetrical profiles due to pressure and resonance broadening. In addition, if the equation of transfer can be integrated for lines which do not show detailed balancing, then the line profile should yield the atomic population in the various levels. In stellar atmospheres there are relatively few lines whose profiles, as determined with the dispersions ordinarily available, are not almost wholly instrumental, and the study of the few non-instrumental profiles is much hampered because they arise from the integrated radiation of the whole stellar disc. The recent analysis of Carroll $\S$ shows, however, that rotationally broadened

* P. ten Bruggencate, $Z s, f . A p . \mathbf{1}, 275$, I93o.

$\dagger$ P. ten Bruggencate, $Z s . f . A p .4$, I $59,1932$.

‡ A. Pannekoek, M.N.R.A.S., 91, I39, I930. § J. A. Carroll, M.N. 93, 478, 508, 680, 1933. 
profiles can be treated in such a way that observational errors are relatively insignificant, and that from these profiles not only the rotational velocity but also a rough estimate of the darkening towards the limb in the stellar disc may be found.

The study of equivalent breadths, a relatively simple spectrophotometric problem, though not without its own difficulties, may be said to have been initiated by the Russell-Adams calibration of the Rowland intensity estimates. Its subsequent development, due largely to Minnaert* and his collaborators, has resulted in the discovery that the equivalent breadth is a somewhat complicated function of the number of atoms in the line of sight, this function known as the "curve of growth" consisting of one branch where Doppler effect predominates and the breadth increases linearly with the number of atoms, joined by a curve almost independent of numbers of atoms to another branch, where the natural line broadening pre dominates, and the breadth varies as the square root of the number of atoms. This curve of growth, surprisingly insensitive to the mode of formation of the absorption line, is found to apply not only to solar but also to stellar absorption lines $\dagger$; recently Struve and Elvey $\neq$ have made the interesting discovery that the curve varies from star to star, and have suggested that this is due to varying amounts of turbulence. Marked though the progress has been, there remain several uninterpreted anomalies, conspicuous among which is the fact that greatly increased damping factors have to be assumed to obtain a fit with the observed curves of growth, the existence of multiplets, as shown by Woolley and Thackeray $\S$, which cannot be fitted into this scheme, and finally the serious difficulties which Menzel has raised against the interpretation by turbulence $\|$.

It is becoming clear that the ultimate solution of the whole problem will require laboratory intensities for multiplet lines, equivalent breadths for these same lines in the stellar atmosphere concerned, and a direct integration, as in the original Russell-Adams calibration, to find the curve of growth. Such a procedure would not only avoid an appeal to uncertain theoretical intensities of lines within multiplets, or from multiplet to multiplet, but also would avoid the use of the irrevelant and inaccurate Rowland intensity estimates. In addition, in the case of the sun, the curve of growth should be determinable from the change in the equivalent breadths of a few lines as observed across the solar disc. If these independent methods yield consistent curves of growth it should then be possible satisfactorily to interpret any remaining anomalies, and to use the curve thus derived to find the atomic population in the various atomic levels.

2.2I. Emission line intensities, especially in cases where it is probable that selfabsorption plays a negligible part, may be physically interpreted with considerably greater ease than absorption line profiles or equivalent breadths. The line spectra of the gaseous nebulae, and the broadened lines of Wolf-Rayet stars are examples of such cases. In both cases, application of Zanstra's theory of nebular emission, to the spectrophotometrically determined flux ratio of line to adjacent continuous spectrum, yields what may be termed a "fluorescent temperature" for the exciting star; such temperatures have the happy characteristic of being lower limits to the true temperature. Further the intensity decrement in the Balmer series has a

* Minnaert and Mulders, Zs. f. Ap. 2, 165, r931; Minnaert and Slob, Proc. Acad. Amsterdam, 34, 542, r93 I M. Minnaert, Observatory, 57, 328, I934.

$\dagger$ T. Dunham, Pub. Amer. Astr. Soc. 7, 2 I 5, 1933.

Struve and Elvey, $A p . J \cdot \mathbf{7 9}, 409$, I934.

$\S$ R. v. d. R. Woolley, Annals S.P.O., Cambridge, 3, 79, I933; A. D. Thackeray, M.N. 94, 99, 1933 .

\|I D. H. Menzel, Pub. A.S.P. 46, 216, 1934. 
simple theoretical relation to the velocity distribution of the captured free electrons*. More difficult of interpretation are chromospheric emission intensities where self-absorption undoubtedly plays an important part; in this field not only observations of the eclipsed and uneclipsed sun are needed, but also an adequate theory of the formation of the emission lines.

\section{SPECTROPHOTOMETRIC TECHNIQUE}

3. Spectrophotometric technique is concerned in the main with the problems of calibration and standardization. Calibration may be defined to be the determination of the response of the photometric receiver as a function of the flux within the wavelength interval $\lambda$ to $\lambda+d \lambda$ incident upon it. Standardization may be defined to be the determination of the fraction of the incident flux to which the spectrophotometer (spectroscope plus photometric receiver) responds as a function of wavelength.

3.I. Calibration. Calibration of the photometric receiver is carried out by varying the flux $T_{\lambda} E_{\lambda}$, where $E_{\lambda}$ is the undetermined flux incident on the receiver when $T_{\lambda}$ is unity, and $T_{\lambda}$ is a known factor which may be varied between zero and unity. Such variation may be carried out by the inverse square law, by diaphragms, polarization methods and so on; it is desirable, but not necessary, that $T_{\lambda}$ should be independent of wave-length. Corresponding to any value of $T_{\lambda}$ there is a definite response, $z$, of the receiver. The object of the calibration is then to find the functional relation

$$
T_{\lambda} E_{\lambda}=f(z)
$$

This relation, frequently shown graphically (the characteristic curve), suffices without a subsequent standardization in those spectrophotometric problems, such as the determination of line profiles, where the ratio of fluxes at one wave-length, or within a very small wave-length interval, alone is required.

Calibration is not only an essential part of spectrophotometric technique, but it also affords a convenient means of classifying photometric receivers according to their sensitivity limit and their inertia. The sensitivity limit may be defined to be the smallest fractional increment in the incident flux, $\Delta f(z) / f(z)$, which is detectable by the receiver; thus the smaller the sensitivity limit the smaller the accidental error of a spectrophotometric determination. From equation (8) and the mean value theorem we find immediately

$$
\frac{\Delta f(z)}{f(z)}=\frac{\mathrm{I}}{f(z)} \frac{d f(z)}{d z} \Delta z=\frac{\mathrm{I}}{T_{\lambda}} \frac{d T_{\lambda}}{d z} \Delta z
$$

from which it is seen that, for a given value of $\Delta z$ (the smallest difference in response which can be measured) the sensitivity limit will vary directly with the slope of the characteristic curve, and inversely as $T_{\lambda}$. Apart from those receivers with linear characteristic curves it is therefore desirable that observation should always be carried out in the range where the slope $d T_{\lambda} / d z$ of the characteristic curve is a minimum. The inertia of a photometric receiver is that flux corresponding to which the receiver makes some definite response; it may be defined as the intercept on the $T_{\lambda} E_{\lambda}$ axis of the tangent to the linear part of the characteristic curve and its

* G. Cillié, M.N. 92, 820, I932. 
evaluation requires a determination of $E_{\lambda}$, a quantity the numerical value of which is not needed in ordinary spectrophotometric work.

The following table lists the more commonly used photometric receivers in order of increasing inertia, giving for each, as well as can be estimated, the optimum sensitivity limit.

\section{Typical Photometric Receivers}

Receiver

Geiger-Müller photon counter(1)

Inertia

ergs. cm.

sec. $^{-1}$

Human eye (2)

$K-H$ photoelectric cell with Hoffman

electrometer (3)

$C s-O-A g$ vacuum photoelectric cell, valve

amplification (4)

Vacuum thermocouple, or bolometer with

thermorelay (5)

Average photographic plate(6)

$8.9 \times 10^{-11}$

$3.7 \times 10^{-9}$

$4.5 \times 10^{-8}$

Optimum sensi-

tivity limit

$\%$

$>1$

0.3

$\sim 1$

$9 \times 10^{-6} \quad \sim 1$

$5 \times 10^{-3} \quad 0 \cdot 1$

$2.6 \times 10^{-2}$

$\mathbf{3} \cdot \mathbf{5}$

(I) B. Rajewsky, Phys. Zs. 32, I21, I931; G. L. Locher, Phys. Rev. 42, 525, 1932.

(2) P. Reeves, $A p . J .46,167,1917 ; 47,14 \mathrm{r}$, 1918. Area pupil $=0.54 \mathrm{~cm}^{2} ; \mathrm{J}$. W. T. Walsh, Photometry, Chap. 6.

(3) Sinclair Smith, Ap.J. 76, 286, r932; Phys. Rev. 43, 2 II, 1933.

(4) F. Löhle, Zs. f. Ap. 6, 293, 1933.

(5) Ornstein, Moll, Burger, Objektive Photometrie, pp. 9, I8, 35.

(6) M. Biltz, Phys. Zs. 34, 200, 1933. For three hour exposure this inertia, applicable to blue region, should be reduced by factor $10^{-4}$.

3.2. Standardization. Standardization is made necessary by the fact that the transmission, $\beta_{\lambda}$, of the spectroscope, and $w_{\lambda}$, the weighting factor which the receiver assigns to the flux to which it responds, must be known. Standardization, except in those cases where $w_{\lambda}$ is known, is only possible through the intermediary of a source of known energy distribution. Assume such a source to be available, and let the flux incident per unit area on the spectroscope slit from it be $\phi_{\lambda}^{\prime} d \lambda$. Then of this incident flux the receiver can only respond to $\beta_{\lambda} w_{\lambda} \phi_{\lambda}^{\prime} d \lambda$; let its response be $z^{\prime}$. Of the unknown flux incident per unit area on the slit, $\phi_{\lambda} d \lambda$ (cf. equation $\mathrm{I}$ ), the receiver responds to the amount $\beta_{\lambda} w_{\lambda} \phi_{\lambda} d \lambda$, the response being $z$. From the calibration we know that to response $z$ corresponds an incident flux $f(z)=T_{\lambda} E_{\lambda}$. Hence, taking the fluxes from the characteristic curve corresponding to $z$ and $z^{\prime}$, and taking their ratio, we obtain

$$
\frac{T_{\lambda}}{T_{\lambda}^{\prime}}=\frac{\beta_{\lambda} w_{\lambda} \phi_{\lambda} d \lambda}{\beta_{\lambda} w_{\lambda} \phi_{\lambda}^{\prime} d \lambda} \text {, or } \phi_{\lambda}=\frac{T_{\lambda}}{T_{\lambda}^{\prime}} \phi_{\lambda}^{\prime},
$$

where $T_{\lambda} / T_{\lambda}^{\prime}$ is known from the calibration, and $\phi_{\lambda}^{\prime}$ is the known flux from the standard source.

Standardization thus requires a source of known energy distribution at least, and, in many problems, of known surface brightness. Apart from laboratory black bodies, the most satisfactory existing standard sources are tungsten filament lamps whose colour and brightness temperatures have been determined at a standardizing laboratory, either with reference to a black body maintained there, or by means of a thermocouple with a strictly linear characteristic curve for which $w_{\lambda}=I$. Filament lamps so standardized have now been subjected to laboratory intercomparisons over a term of years*, and the small differences still outstanding between different

* W. E. Forsythe, Phys. Rev. 38, 1247, 1931. 
laboratories appear likely to be removed by recent work on the freezing point of platinum*. It cannot, however, be maintained that such filament lamps are the ultimately desirable standard sources; they are unsatisfactory partly because the method of colour matching generally used only leads to an energy distribution known in the range $\lambda \lambda_{4000-7000}$ to an accuracy of about I per cent., and partly because, even when under-burned, their life, before re-standardization becomes necessary, is only the order of one hundred hours. The ideal standard would of course be a reproducible standard, such as before the Greenwich investigation the acetylene flame was believed to be†, the energy distribution of which should be known at least over the range $\lambda \lambda 3000-7000$. It is possible that such a source has been found in the hydrogen discharge tube investigated by Chalonge and his colleaguest, though the well-known dependency of gaseous discharges on electrodes and form of tube will demand an extended series of investigations to establish the reproducibility of this source.

3.21. Substitution Method. Sometimes of necessity, when for example the photometric receiver is the human eye, and sometimes by preference, calibration and standardization are reduced to one operation by the substitution method. In this method the flux from the standard source, $\beta_{\lambda} w_{\lambda} \phi_{\lambda}^{\prime} d \lambda$, incident on the receiver, is changed in some suitable way by the factor $\gamma$ until the response $z^{\prime}$ for the standard source is identical with the response $z$ for the unknown source. We then have

$$
\gamma \beta_{\lambda} w_{\lambda} \phi_{\lambda}^{\prime} d \lambda=\beta_{\lambda} w_{\lambda} \phi_{\lambda} d \lambda \text {, or } \phi_{\lambda}=\gamma \phi_{\lambda}^{\prime} \text {. }
$$

This substitution method is also of course applicable in those cases where only calibration is needed, as for example in the determination of line profiles. The substitution method has the great advantage that variability in the characteristic curve of the receiver, a source of difficulty in sensitized photoelectric cells and in photographic plates, is as a source of systematic error almost wholly eliminated. In using the substitution method, however, care should be taken that the substitution is performed at a response $z$ where $d T_{\lambda} / d z$ is as small as possible, otherwise, as equation (9) shows, the sensitivity limit, and correspondingly the accidental error will be greatly increased. Also it should be noted that accidental error will be increased by this method if the method of varying $\gamma$ has not as small a sensitivity limit as that of the receiver.

3.3. Recent Developments in Technique. There can be little doubt that the new methods of using the external photoelectric effect for the construction of sensitive and stable photometric receivers constitute the most significant recent development in spectrophotometric technique. Though it is a misnomer to call the Rajewsky and Locher cells photon counters, since the most highly sensitized surface available yields but one electron for $10^{3}$ incident quanta, yet the discovery that the addition of a photosensitive surface and a quartz window to a Geiger-Müller counter would enable each photoelectrically ejected electron to be counted is of the first importance in astronomical spectrophotometry. It would of course be anticipated that such counters would show as photometric receivers the well-known defects of the gasfilled cell in an exaggerated form, namely its instability, its time lag, and its fatigue and recovery effects. The surprising result is that Locher finds, in spite of these defects, a sensitivity limit of I.8 per cent. for a properly designed counter. An im-

* B.S. Research Paper, 6, No. 325, r931; N.P.L. Report r933, pp. 28, 91, 92.

$\dagger$ R.O., Greenwich, Colour Temperatures, p. 32, 1932.

$\ddagger$ D. Chalonge, Ann. de Phys. I, 123, I934. 
portant application of this receiver has recently been made by Meyer, Schein and Stoll* to the measurement of the solar radiation transmitted between the ozone and oxygen bands at $\lambda 2100$.

Possibly more significant than the development of receivers with small inertia has been the use of the vacuum photoelectric cell, which when properly designed and constructed is, as regards linearity of characteristic curve and smallness of sensitivity limit, nearly the ideal photometric receiver. Noteworthy in this field has been the application of a Hoffmann electrometer by Sinclair Smith, and several applications of D.C. amplifiers such as the FP-54 Pliotron. In spectrophotometry Dunham $\dagger$ has made a pioneer determination of solar line profiles with a vacuum $K-H$ cell connected directly to the grid of a Pliotron, the cell, amplifier and leads being enclosed in an evacuated cylinder to minimize external ions. More recently he has used the substitution method, matching the flux from the adjacent continuous spectrum, reduced by a polarization device, with the flux from a wave-length interval of 0.03 A within the line. His sensitivity limit is well under one per cent.

3.3I. A spectrophotometric problem, which will necessarily bulk larger as objective receivers, such as the photoelectric cell and the thermocouple, are substituted for the photographic plate, is the reduction of scattered light in the spectroscope. Early trials of a monochromator for this purpose were made by Dunham + with the I50-foot Tower Telescope at Mt Wilson in I93I, but the most successful treatment of the problem to date has been Redman's $\S$ design of a double monochromator. A simple method of securing this preliminary analysis, now in use by Dunham at Mt Wilson, is afforded by a Christiansen filter\|; this filter, which consists of powdered quartz in a mixture of benzole with bisulphide of carbon, transmits a band some I5 A wide at the wave-length where the refractive index of powder and liquid is the same. The wave-length of the transmitted band can be changed by heating the filter.

3.32. As reference to the table in $\S 3$. I shows, the human eye is, as regards its inertia and sensitivity limit, the most satisfactory of receivers. Its only limitation, and that not a serious one, is that it must be used in the substitution method. It is therefore somewhat surprising that, apart from the pioneer investigations of Wilsing and Scheiner, the eye has been so little used in astronomical spectrophotometry, the more so when it is recalled how important is the part played by visual spectrophotometry in standardizing laboratories. As has long been recognized, the principle of the Maxwellian view is fully applicable to stellar photometry and spectrophotometry, and in an interesting paper Schering** has given a simple design for a visual photometer, changeable at will to a spectrophotometer, which may be used with an astronomical telescope. A modification of Schering's design has recently been applied by Hopmann $\uparrow \dagger$ to the construction of a visual photometer (not spectrophotometer), and with it he has already secured some interesting results.

3.33. In spite of its well-known defects (see table in $\S 3 \cdot I$ ), it is probable that the photographic plate will retain for some years to come its present pre-eminent position as a photometric receiver in astronomical spectrophotometry. This position is due not entirely to the simplicity of its use, but partly to the fact that it permits

* Meyer, Schein, and Stoll, Nature, 134, 535, I934.

$\dagger$ T. Dunham, Jr., Phys. Rev. 44, 329, r933.

$\ddagger$ Year Book, Carnegie Institution, 30, I94, 1931. $\$$ R. O. Redman, M.N. 95, 290, 1935.

if Rayleigh, Collected Papers, 2, 433, I885; 4, 392, 1899.

I J. W. T. Walsh, Photometry, pp. 399, 425. ** H. Schering, Phys. Zs. 22, 71, 1921.

†† J. Hopmann, Leipzig Publications, Part III, r932. 
the simultaneous recording of widely separated spectral regions, a valuable property when the variability of atmospheric transmission is recalled, and partly to the fact that it permits a simple elimination of light scattered in the spectroscope (apart from that produced by grating ghosts). It is therefore highly desirable that attention should again be directed to three difficulties which, if eliminated, would put photographic spectrophotometry on a completely different footing. These difficulties are first the large sensitivity limit, secondly Eberhard and scattering effects in the emulsion, and finally the time-consuming nature of the reduction of photographic densities to incident fluxes.

Of these difficulties by far the most important is the first. Undoubtedly part of the large sensitivity limit of 3.5 per cent. arises from a combination of effects which are just on the limit of detectability, such as the fading of the latent image with time, the variation of density for a constant incident flux as a function of the hygroscopic condition and temperature of the emulsion, and the abnormal effect noted by Ross*. These can all be eliminated, however, by a simultaneous exposure of the calibrating source with the unknown or the standardizing source, and when they are so eliminated there is still left a sensitivity limit of the order of three per cent. Carroll $\uparrow$ has recently made the interesting suggestion that this residual limit is due to variations of thickness or sensitivity of the emulsion with an irregular wave-length of about a quarter of a mm. Experiments in progress at Aberdeen suggest that, if precautions are taken to make the emulsion of uniform thickness, the sensitivity limit is reduced. If confirmed, this is a most important result, since some simple modifications in the manufacture of photographic plates for photometric use might greatly decrease the sensitivity limit. Further experiments along similar lines are greatly to be desired.

The second difficulty, due to the failure of the plate properly to register densities over small areas, may be greatly reduced in effect by procedures suggested by

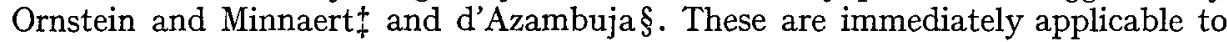
the determination of line profiles, the problem where this failure produces the most serious errors. Ornstein and Minnaert make use of an enlarging camera, whereas d'Azambuja photographs the disc of the sun with the second slit of his spectroheliograph set on the desired wave-length interval in the absorption line.

The third difficulty, while not a defect of the photographic plate as a photometric receiver, is yet an obstacle to the rapid and accurate reduction of spectrophotometric observations. The difficulty occurs in its most exaggerated form when the plates are measured with a recording microphotometer, and the various methods which have been devised by Wouda $\|$, by Dunham $\rrbracket$, by Thompson**, and by Langstroth $\uparrow \uparrow$ to overcome the difficulty all refer to records made with such a microphotometer. From the published descriptions it would appear that Wouda's Wheatstone bridge method is the neatest of these, but even it will only effect a considerable saving of time when many measurements have to be reduced with respect to the one characteristic curve.

3.34. There remains one aspect of spectrophotometric technique where progress

* F. E. Ross, $A p . J$. 73, 54, I931.

$\dagger$ J. A. Carroll, M.N. 93, 686, r933.

$\$$ Ornstein and Minnaert, B.A.N. 5, 175, 1930 .

$\S$ L. d'Azambuja, Meudon Publications, 8, Part II, p. 65, I930.

II J. Wouda, Zs. f. Ph. 79, $5^{\text {II }, ~ 1932 . ~}$

T T. Dunham, Jr., Pub. Am. Ast. Soc. 7, 215, 1933.

** N. Thompson, Proc. Phys. Soc. (London), 45, 44I, 1933.

†† G. O. Langstroth, Rev. Se. Inst. 5, 255, 1934. 
has been on the whole disappointing, namely in the methods of varying the incident flux by a known amount $T_{\lambda}$ as required in the determination of characteristic curves. No essentially new methods have been recently proposed, though to Ohman* is due a most ingenious application of the polarization produced by reflection off the prism faces, and to Dunham $\uparrow$ is due an application of the variable slit width method, which permits the simultaneous registration of the calibration exposures with that of the unknown and standard sources. As against these advances must be set, however, van Cittert'sł demonstration of the unsatisfactory nature of the "raster" when applied to spectroscopes, and the fact that reflection from the highly reflecting metallic step wedge necessitates a separate determination of its transmissions for each spectroscope with which it is used. A question still unsettled in this field is the range of applicability of the rotating sector as applied to photographic plates and photo-electric cells. The recent work of Webb§ would appear to indicate that after a certain flash frequency is reached, corresponding to the passage of a single quantum, the rotating sector no longer shows the intermittency effect with the photographic plate. This demonstration appears, however, neither more nor less convincing than other demonstrations which have given very different results; especially it should be emphasized that the simple theoretical interpretation which can be given Webb's result is no guarantee that the rotating sector affords a satisfactory method of calibration for photographic plates. Applied to photoelectric cells, however, there seems to be a greater measure of unanimity $\|$ that the rotating sector obeys Talbot's law, though some further work might not be undesirable.

In view of the difficulty of finding wholly satisfactory methods of calibration it is desirable to draw attention to three suggestions which have been made for a cooperative study and comparison of the methods employed. Perhaps the most useful of these is a suggestion of Minnaert in 1935 that a number of solar lines should be selected as standards, and that the profiles of these lines should be determined at a number of separate institutions. The second suggestion, closely related to this, is that of Beals in I933 that the profiles of a number of stellar lines should be similarly determined. Finally there is the suggestion that the Commission should make itself responsible for securing a number of neutral tint filters, the transmissions of which at a number of wave-lengths would be determined at several standardizing laboratories; these filters could then be loaned to any institution wishing to test its method of calibration.

\section{CoNCLUSION}

This report has passed in review first the unique character of spectrophotometric measures in astronomical studies of radiation, secondly the various fields where spectrophotometry has an important part to play, and finally various problems of technique. The Commission will have fully served itspurpose if it affords means and an opportunity for the discussion of these and related questions by its members.

\footnotetext{
H. H. Plaskett

President of the Commission.

April 4, I935.

* Y. Öhman, Stockholms Medd. No. 12, 1933.

$\dagger$ T. Dunham, Jr., Pub. Am. Ast. Soc. 7, 215, 1933.

$\ddagger$ P. H. van Cittert, $Z s . f . P h .73,249$, I93I.

$\S$ J. H. Webb, J. Opt. Soc. Am. 23, I $57,1933$.

II Campbell and Ritchie, Photoelectric Cells, p. 194, 1934.
} 